1155

\section{SCREENING AND TREATMENT STRATEGIES FOR METABOLIC BONE DISEASE IN PRETERM INFANTS: RESULTS OF A NATIONAL QUESTIONNAIRE SURVEY IN THE NETHERLANDS}

\author{
F. Visser, A.J. Sprij, F. Brus \\ Paediatrics, Juliana Children's Hospital, Haga \\ Hospital, The Hague, The Netherlands
}

Background and aims: Consensus about screening and treatment of metabolic bone disease (MBD) in preterm infants is lacking. Aim is to study screening methods and treatment strategies for MBD in preterm infants in The Netherlands.

Methods: Aquestionnaire was sent to all 96 hospitalbased paediatric practices in The Netherlands. Practices were asked whether preterm infants are screened for MBD. Criteria for screening, use of serum and/or urine measurements and imaging techniques were questioned, as were postnatal time points of screening, starting of treatment and type of treatment.

Results: A response rate of $86.5 \%$ was achieved. Commonly applied screening methods are serum concentrations of alkaline phosphatase (serum AP, $73.5 \%$ ), calcium and phosphate (both 61.4\%). Urinary calcium and phosphate concentrations are less frequently applied (36.1\%). Different normative serum and urine values are used. X-ray is mainly performed if abnormal biochemical results occur. Dual energy X-ray absorptiometry (DEXA) and quantitative ultrasound (QUS) are hardly applied as screening methods. Gestational age is the most frequently applied indication for screening of MBD. There is a wide variation of postnatal screening moments. Treatment strategies differ considerably and consist of (combinations of) mineral and/or vitamin D supply.

Conclusions: Screening procedures and treatment strategies for MBD in preterm infants vary considerably, probably reflecting lack of evidence or conflicting evidence. The usefulness of biochemical measurements and/or imaging techniques as screening tools for MBD in preterm infants needs to be clarified as well as the frequency and postnatal time points of screening and strategies for prevention and treatment of MBD.
SCREENING FOR GLUCOSE-6-PHOSPHATE DEHYDROGENASE (G6PD) DEFICIENCY IN NEONATES: A COMPARISON BETWEEN CORD AND PERIPHERAL BLOOD SAMPLES

M.B.P. Ponferrada ${ }^{1}$, S. Al-Saif ${ }^{1}$, K. Khairy², K. Tawil ${ }^{1}$, I. Hakim ${ }^{1}$, H. Tawakol ${ }^{1}$, A. Sallam ${ }^{1}$, K. Hathlol ${ }^{1}$, B. Baylon ${ }^{1}$, V. Manzano ${ }^{1}$

${ }^{1}$ Paediatric-Neonatology, ${ }^{2}$ Pathology and Laboratory Medicine, National Guard Health Affairs, Riyadh, Saudi Arabia

Background and aims: The use of cord blood in the neonatal screening for G6PD deficiency is convenient but has yet to be adequately evaluated against the use of peripheral sample. We sought to determine the incidence and gender distribution of G6PD deficiency in our institution and compare the use of cord against peripheral blood in the identification of G6PDD neonates using quantitative enzyme activity assay.

Methods: We carried out a retrospective, crosssectional, and crossover study employing review of primary hospital data of neonates born from January to December 2008.

Results: Among the 8,139 neonates with cord blood G6PD assays taken, an overall incidence of $2 \%$ for G6PD deficiency was computed. $79 \%$ of these are males and $21 \%$ are females with significantly lower values in deficient males $(p<0.001)$. Gender-specific incidence is $3 \%$ for males and $0.84 \%$ for females. A subgroup analysis comparing cord and peripheral blood samples $(n=1,253)$ showed a significantly higher mean G6PD value for peripheral than cord blood $(15.12 \pm 9.04 \mathrm{U} / \mathrm{gHb}$ and $14.52 \pm 8.86 \mathrm{U} / \mathrm{gHb}$, respectively, $p=0.0008$ ). However, the proportion of G6PD deficient neonates did not significantly differ in the two groups $(p=0.79)$. Sensitivity of cord blood in screening for G6PD deficiency was $98.6 \%$ with a NPV of $99.5 \%$.

Conclusion: There is no difference between cord and peripheral blood samples in detecting G6PD deficiency. The significantly higher mean peripheral G6PD assay reinforces the use of cord blood for G6PDD screening since it has substantially low false negative results. Potential reasons for the trends were explored. 